\title{
铼催化的醇脱羟基反应研究进展
}

\author{
毛国梁*,a 贾 冰 ${ }^{a, b}$ 王从洋 $*, b$ \\ ( ${ }^{a}$ 石油与天然气化工省重点实验室 东北石油大学化学化工学院 大庆 163318) \\ ( ${ }^{b}$ 北京分子科学国家实验室 中国科学院分子识别与功能重点实验室 中国科学院化学研究所 北京 100190)
}

\begin{abstract}
摘要 近年来, 发展将丰富的可再生资源转化成燃料和高附加值化学品的新型高效方法引起人们的广泛关注. 其中的 研究热点之一是多元醇和碳水化合物的选择性化学转换. 多元醇及碳水化合物富含氧元素, 其很大部分是以羟基官能 团的形式存在，因此发展选择性的脱羟基反应具有重要的意义. 介绍了近年来钭催化的醇脱羟基反应的研究进展，主 要分为两部分: (1)单醇的脱羟基反应构建新的化学键; (2)二(多元)醇的脱羟基反应生成烯烃.
\end{abstract}

关键词 铼催化; 醇; 脱羟基; 还原剂; 烯烃

\section{Recent Progress in Re-Catalyzed Dehydroxylation Reactions}

\author{
Mao, Guoliang*,a Jia, Bing ${ }^{a, b} \quad$ Wang, Congyang*,b \\ $\left({ }^{a}\right.$ Provincial Key Laboratory of Oil \& Gas Chemical Technology, College of Chemistry and Chemical Engineering, \\ Northeast Petroleum University, Daqing 163318) \\ ( ${ }^{b}$ Beijing National Laboratory for Molecular Sciences, CAS Key Laboratory of Molecular Recognition and Function, \\ Institute of Chemistry, Chinese Academy of Sciences, Beijing 100190)
}

\begin{abstract}
The development of novel effective processes for the conversion of abundant renewable resources to fuels and value-added chemicals has spurred new interest in the discovery of selective chemical transformations of polyols and carbohydrates. These compounds have high oxygen content, which is mostly present in the form of hydroxyl groups. Therefore, partial or complete removal of the hydroxyl groups is of great importance. Herein a brief overview on the development of rhenium-catalyzed dehydroxylation reactions in recent years is given, which are roughly classified into two categories: (1) dehydroxylation reactions of monools for new chemical bond formation and (2) dehydroxylation reactions of diols and polyols for olefin syntheses.
\end{abstract}

Keywords rhenium catalysis; alcohols; dehydroxylation; reductants; olefins

当前, 绝大多数的化学工业使用矿物燃料. 随着矿 物资源的日渐减少，寻找替代资源刻不容缓. 生物资源 作为一种可再生资源有潜力成为化学工业中矿物燃料 的一种替代资源, 生物资源每年能够向化学工业提供大 量的碳源基础原料 ${ }^{[1]}$, 然而生物资源的组成与矿物燃料 资源有很大的不同, 生物资源富含氧元素, 很大部分是 以羟基官能团的形式存在. 将生物资源转化成化学工业 及其他领域可利用的燃料, 需要将生物资源中的羟基官 能团移除或转化(脱羟基反应), 使其氧碳比下降 ${ }^{[2 \sim 4]}$.

脱羟基反应目前大多使用强酸催化剂体系, 例如使
用硫酸、对甲苯磺酸等液体酸; 或者沸石、氧化铝、氧 化锆等固体酸. 使用这些方法的最主要问题是反应体系 的选择性及官能团容忍性低, 并且由于酸的自身性质存 在腐蚀及安全等因素. 铼催化体系在脱羟基反应中选择 性好, 反应条件相对温和, 反应产率高, 引起了化学家 们的广泛兴趣. 因此, 铼催化的醇脱羟基反应近年来取 得了重要的进展. 基于对铼催化有机转化的研究兴趣 ${ }^{[5]}$, 我们主要从两个方面介绍铼催化的醇脱羟基反应的研 究进展: (1)单醇的脱羟基反应构建新的化学键; (2)二(多 元)醇的脱羟基反应生成烯烃.

*E-mail:mglww001@yahoo.com.cn; wangcy@iccas.ac.cn

Received September 11, 2014; revised November 11, 2014; published online November 20, 2014.

Project supported by the Scientific Research Foundation for Overseas Chinese Scholars of Heilongjiang Province (No. 1154H14), the Department of Education of Heilongjiang Province (No. 1154G53), the Joint Funds of National Natural Science Foundation of China (No. U1362110), and the National Natural Science Foundation of China (Nos. 21322203, 21272238).

黑龙江省教育厅海外学人科研(No. 1154H14)、黑龙江省教育厅(No. 1154G53)、国家自然科学基金联合基金(No. U1362110)、国家自然科学基金(Nos. 21322203, 21272238) 资助项目. 


\section{1 锞催化单醇脱羟基反应构建新的化学键}

1996 年, Espenson 课题组 ${ }^{[6]}$ 首次报道了 $\mathrm{MeReO}_{3}$ 催 化单醇的脱羟基反应. 该反应包括四个方面的内容 (Scheme 1): (1) $\mathrm{MeReO}_{3}$ 催化单醇生成醚. 反应使用 $\mathrm{MeReO}_{3}$ 催化剂, 以苯作为溶剂, 在室温下反应 $2 \mathrm{~d}$, 两 分子醇脱水生成醚. 带有芳香官能团的仲醇脱羟基生成 醚具有优秀的收率. 该体系也适用于两个不同单醇的交 叉反应, 生成不对称的醚. 控制生成不对称醚的重要因 素是使反应活性高、带有芳香官能团的醇的浓度低于带 有脂肪官能团的醇, 虽然反应中有对称的醚生成, 但是 不对称的醚是主要产物. 如果两个单醇具有相似的反应 活性, 则生成产率相近的三种醚的混合物. (2) $\mathrm{MeReO}_{3}$ 催化单醇脱羟基生成烯烃. 脂肪醇生成烯烃的反应只有 中等收率, 而芳香醇生成烯烃的反应则能取得满意的结 果. 作者在文中没有明确指出单醇脱羟基生成烯烃的机 理, 我们推测反应可能是经过铼氧物种协同脱水机制 (经由 Scheme 1 中间体 $\mathbf{A}$ )或通过碳正离子机制(经由 Scheme 1 中间体 C). (3) $\mathrm{MeReO}_{3}$ 催化芳香伯醇与伯胺 的氨基化反应. 在醇过量的条件下, 该反应对于合成仲 胺化合物具有优秀的产率, 反应过程中同时有醇脱水产 物一一醚的生成, 当醇与胺按当量比例反应时, 反应产 物仲胺的产率会下降. (4) $\mathrm{MeReO}_{3}$ 催化二芳香醇的歧化 反应生成二芳基酮和二芳基甲烷产物. 除了少数几个醇 以外, 多数醇类化合物在此催化体系中都发现有一定量 的歧化反应发生. 对于反应的机理, 作者推测反应经历

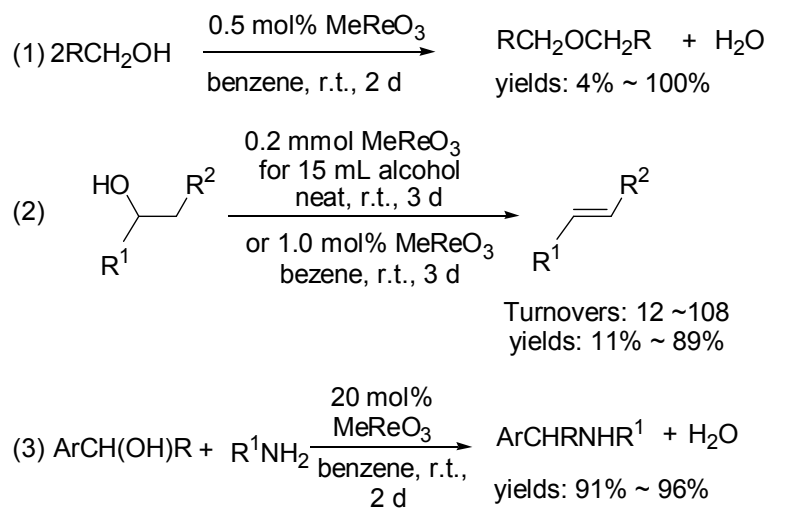

(4) $\mathrm{Ar}^{1} \mathrm{Ar}^{2} \mathrm{CHOH} \frac{2 \mathrm{~mol} \% \mathrm{MeReO}_{3}}{\text { benzene, r.t., } 2 \mathrm{~d}} \mathrm{Ar}^{1} \mathrm{Ar}^{2} \mathrm{CO}+\mathrm{Ar}^{1} \mathrm{CH}_{2} \mathrm{Ar}^{2}$

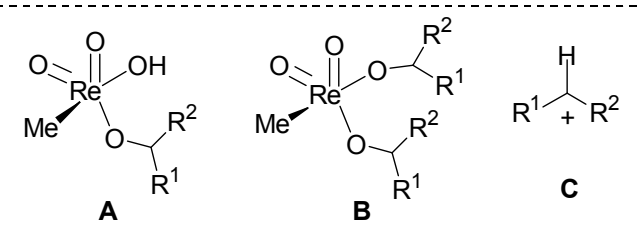

图式 $1 \mathrm{MeReO}_{3}$ 催化的单醇脱羟基反应

Scheme $1 \mathrm{MeReO}_{3}$-catalyzed dehydroxylation reactions of monools
了反应中间体 $\mathbf{A}$, 从 $\mathbf{A}$ 到产物的生成, 可能经历了两种 不同的路径: 一种是协同机制，例如，对于成醚反应， 可能是经过中间体 $\mathbf{B}$ 的协同过程; 另一种是碳正离子机 制(中间体 C). 这两种机制可能在不同的底物反应中分 别起到主导作用.

在 Espenson 课题组工作的基础上, 关于铼催化单 醇脱羟基构筑新化学键的研究逐渐活跃起来, 下文将对 此后铼催化单醇脱羟基构筑 $\mathrm{C}-\mathrm{O} / \mathrm{C}-\mathrm{N} / \mathrm{C}-\mathrm{C}$ 键的反 应进展做简要的介绍.

\section{1 铼催化单醇脱羟基构筑 $\mathrm{C}$ - $\mathrm{O}$ 键反应}

近些年, 钭催化单醇脱羟基构筑 $\mathrm{C}-\mathrm{O}$ 键取得了进 展. 2003 年, Toste 课题组 ${ }^{[7]}$ 报道了铼催化剂(dppm)$\mathrm{ReOCl}_{3}$ 催化的醇和炔丙醇偶联生成 $\mathrm{C}-\mathrm{O}$ 键的反应 (Scheme 2,a). 作者尝试了多种催化剂后发现, 含有双 齿膦配体的铼催化剂对反应最有效. 1-位芳基取代的炔 丙基醇都能较好地与脂肪醇亲核试剂反应生成相应的 醚化合物. 特别指出的是, 1-位对溴苯基取代的炔丙醇 也能与 3-丁烯-1-醇反应生成醚化合物 $(60 \%)$, 产物中的 澳官能团可以使用后过渡金属催化剂进行后续的衍生 化反应. 炔键上含有各种烷基、芳基、三甲基硅基、酯 基取代的底物, 通过适当的升高反应温度及延长反应时 间都能较好地生成醚化合物. 亲核试剂中含有氯官能团 的烷基醇也可以生成相应的醚产物, 并且没有氯消除的 副产物生成. 作为亲核试剂的伯醇与仲醇都能生成相应 的醚，反应效果没有明显差别，但是当以叔丁醇作为亲 核试剂时，生成梄只有 $30 \%$ 的产率.

华瑞茂课题组 ${ }^{[8]}$ 在 2005 年首次报道用低价钭催化 剂 $\operatorname{ReBr}(\mathrm{CO})_{5}$ 催化茮醇与脂肪族醇 $\left(\mathrm{C}_{1} \sim \mathrm{C}_{16}\right)$ 的脱水醚 化反应(Scheme 2, b). 该反应产率高, 化学选择性好, 两种原料醇自身脱水成醚的副产物很少. 作者认为反应 机理如下: (1) $\operatorname{ReBr}(\mathrm{CO})_{5}$ 被氧气氧化生成三价的钭氧化 物; (2)三价的铼氧化物脱羰基生成 16 电子的中间体 $\operatorname{Re}(\mathrm{O}) \mathrm{Br}(\mathrm{CO})_{4} ;$; (3)醇对三价锞氧化物中 $\mathrm{Re}=\mathrm{O}$ 键加成, 然后 $\mathrm{C}-\mathrm{O}$ 键断裂生成芐基正离子, 脂肪醇进攻苄基正 离子形成不对称的醚和含有二羟基的三价铼化合物; (4) 含有二羟基的三价铼化合物脱水生成 $\operatorname{Re}(\mathrm{O}) \operatorname{Br}(\mathrm{CO})_{4}$ 完成催化循环.

\section{2 铼催化单醇脱羟基构筑 $\mathrm{C}-\mathrm{N}$ 键反应}

2005 年, Toste 课题组 ${ }^{[9]}$ 继续用 $(\mathrm{dppm}) \mathrm{ReOCl}_{3}$ 催化 了炔丙醇与磺酰胺和氨基甲酸酯类化合物的偶联反应, 生成了炔丙胺化合物, 发展了一类构筑 $\mathrm{C}-\mathrm{N}$ 键的新方 法(Scheme 3, a). 该反应条件温和, 适用底物范围广, 反 应产率高. 作者进一步将反应体系成功应用于具有生物 活性的含氮杂环化合物 Pentabromopseudlin 的合成中 (Scheme 3, b). 
(a)

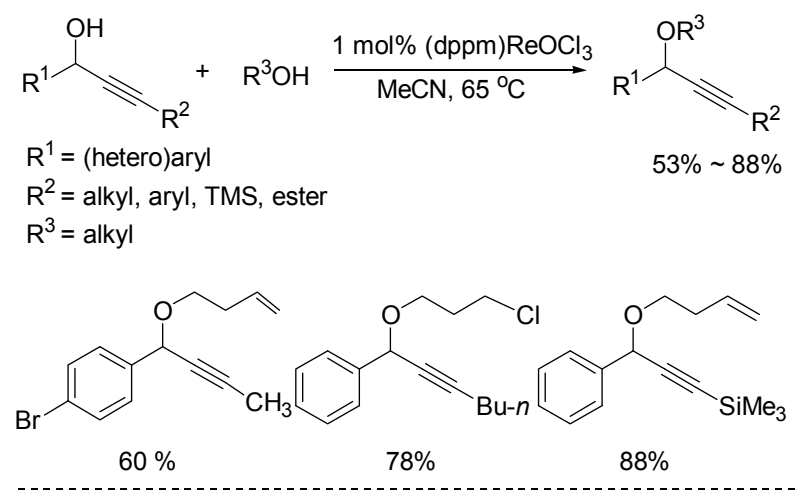

(b)

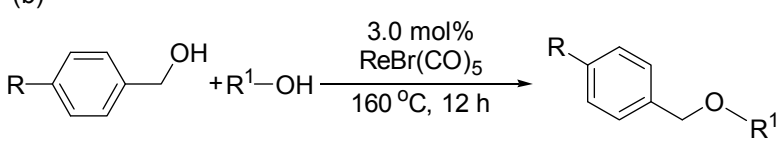

$\mathrm{R}=\mathrm{H}, \mathrm{CH}_{3}, \mathrm{Cl}$

$\mathrm{R}^{1}=\operatorname{alkyl}\left(\mathrm{C}_{1}-\mathrm{C}_{16}\right)$

$60 \% \sim 99 \%$

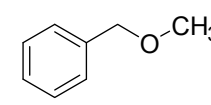

$99 \%$

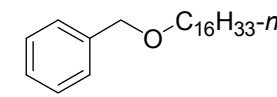

$60 \%$

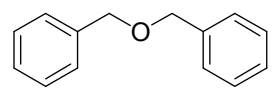

$78 \%$

Proposed Catalytic Cycle

$\operatorname{ReBr}(\mathrm{CO})_{5}+1 / 2 \mathrm{O}_{2} \longrightarrow \mathrm{O}=\operatorname{ReBr}(\mathrm{CO})_{5}$

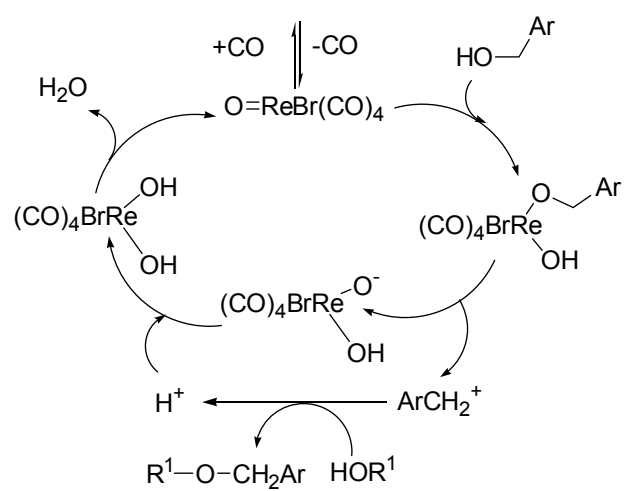

图式 2 铼催化的醇脱羟基反应构建 $\mathrm{C}-\mathrm{O}$ 键

Scheme 2 Re-catalyzed dehydroxylation reactions of alcohols for $\mathrm{C}-\mathrm{O}$ bond formation

2014 年, 朱成建课题组 ${ }^{[10]}$ 报道了 $\left[\mathrm{ReH}_{7}\left(\mathrm{PCy}_{3}\right)_{2}\right]$ 催 化的醇的直接脱羟基胺化反应. 该反应对于醇类底物有 较广泛的应用范围, 适用于一系列的伯醇和仲醇底物 (Scheme 4). 需要指出的是, 反应底物中的胺化试剂为 芳胺化合物, 有一定的局限性. 该反应的优点是反应产 物为单烷基化的苯胺, 没有二烷基化产物的生成; 另外, 反应的副产物是水, 没有其他废弃物的生成, 是一条绿 色合成胺的有效方法. 作者认为反应经历了 “借氢” 还 原胺化 ${ }^{[11]}$ 的机理: 即醇首先在铼催化下脱氢生成醛, 醛 和芳胺去水缩合生成亚胺, 亚胺再被氢化最终得到醇脱 羟基胺化的产物. (a)<smiles>[R]C#CC([R])O</smiles>
$5 \mathrm{~mol} \%(\mathrm{dppm}) \mathrm{ReOCl}_{3} \quad \mathrm{R}^{3}-\mathrm{N}^{-} \mathrm{R}^{4}$ $\mathrm{R}^{1}=$ aryl $\mathrm{R}^{2}=$ alkyl, TMS, alkenyl, ester $5 \mathrm{~mol} \% \mathrm{NH}_{4} \mathrm{PF}_{6}$

$\mathrm{MeCN}$ $\mathrm{R}^{3}, \mathrm{R}^{4}=$ alloc, Boc, Cbz, Ts, H, aryl, alkyl, etc.

(b) Synthesis of pentabromopseudilin

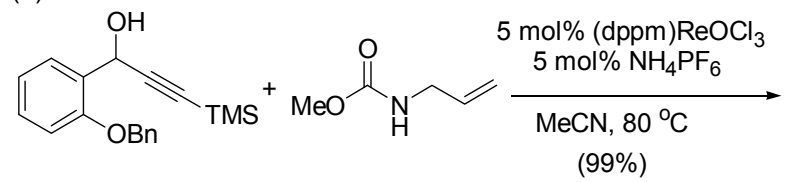

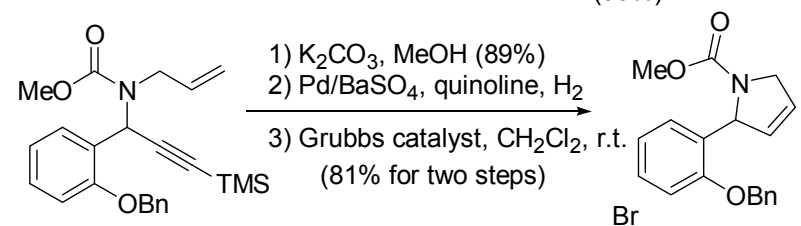

1) $\mathrm{DDQ}, \mathrm{PhMe}(63 \%)$ 2) $\mathrm{NaOH}, \mathrm{EtOH}(95 \%)$

3) $\mathrm{Na}, \mathrm{NH}_{3}(80 \%)$ 4) $\mathrm{PyHBr}_{3}, \mathrm{EtOH}(52 \%)$<smiles>Oc1c(Br)cc(Br)cc1-c1[nH]c(Br)c(Br)c1Br</smiles>

Pentabromopseudilin

图式 3 (Dppm) $\mathrm{ReOCl}_{3}$ 催化的炔丙醇脱羟基反应构建 $\mathrm{C}-\mathrm{N}$ 键

Scheme 3 (Dppm) $\mathrm{ReOCl}_{3}$-catalyzed dehydroxylation reactions of propargylic alcohols for $\mathrm{C}-\mathrm{N}$ bond formation
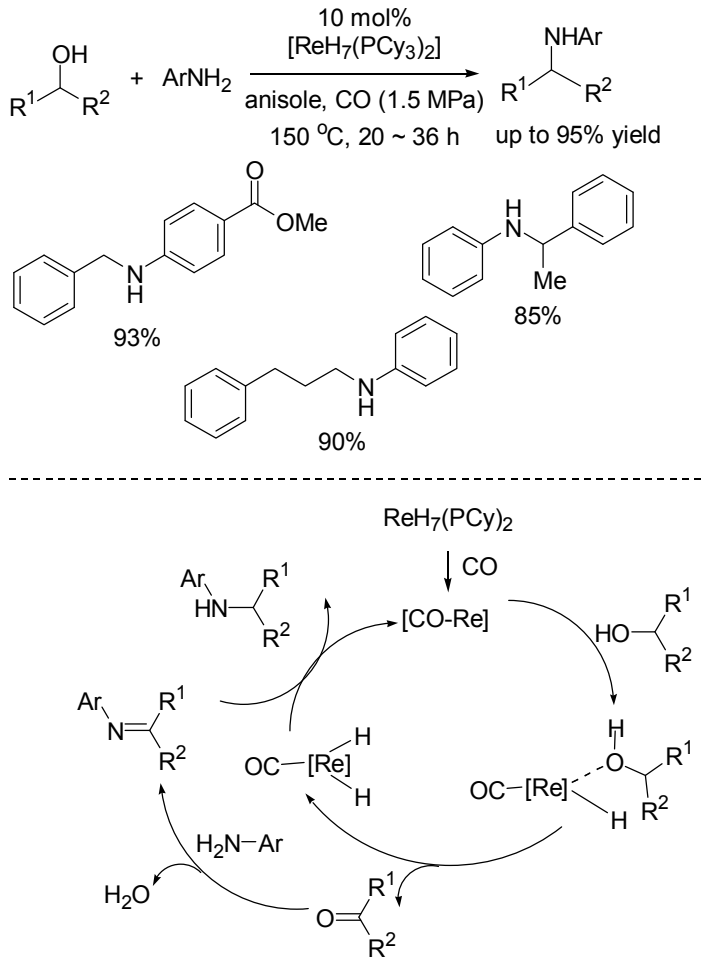

图式 $4 \mathrm{ReH}_{7}(\mathrm{PCy})_{2}$ 催化的醇脱羟基反应构建 $\mathrm{C}-\mathrm{N}$ 键 Scheme $4 \mathrm{ReH}_{7}(\mathrm{PCy})_{2}$-catalyzed dehydroxylation reactions of monools for $\mathrm{C}-\mathrm{N}$ bond formation 


\section{3 铼催化单醇脱羟基构筑新 $\mathrm{C}-\mathrm{C}$ 键反应}

2003 年, Toste 课题组 ${ }^{[12]}$ 报道了铼催化的烯丙基硅 烷与炔丙醇的 $\mathrm{C}-\mathrm{C}$ 键形成反应. 反应中使用的催化剂 (dppm) $\mathrm{ReOCl}_{3}$ 对空气和水分容忍性好, 该方法适用于 制备一系列的 1,5-烯炔, 底物适用范围广, 反应产率高 (Scheme 5,a). 当采用手性的巴豆基硅烷与炔丙醇反应

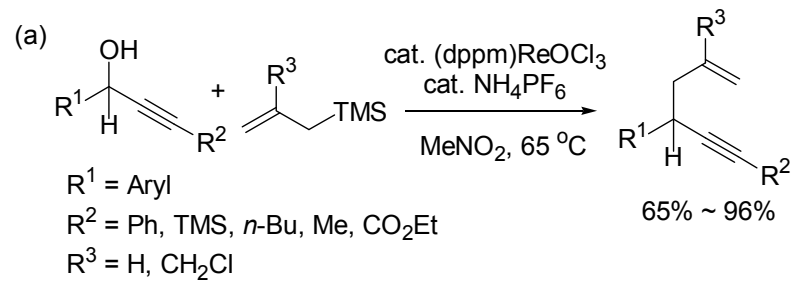

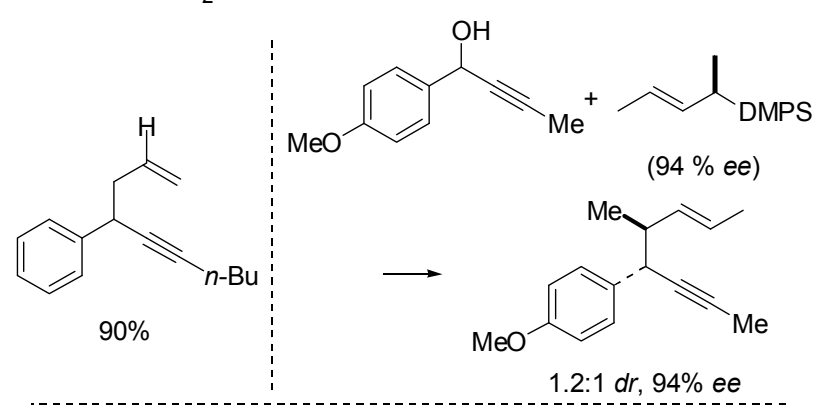

(b)<smiles>C=CCc1ccc2c(c1)OCO2</smiles><smiles>C=CCc1cc2c(cc1C(C#CC(=O)OCC)c1cc(OC)c(OC)c(OC)c1)OCO2</smiles>

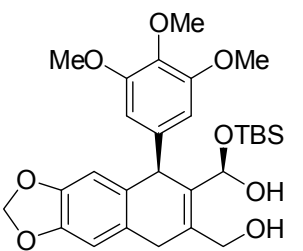<smiles>COc1cc([C@H]2C3=C(COC3=O)Cc3cc4c(cc32)OCO4)cc(OC)c1OC</smiles>

apopicropodophyllin

图式 5 (Dppm) $\mathrm{ReOCl}_{3}$ 催化的脱羟基反应构建 $\mathrm{C}-\mathrm{C}$ 键 Scheme 5 (Dppm) $\mathrm{ReOCl}_{3}$-catalyzed dehydroxylation reactions for $\mathrm{C}-\mathrm{C}$ bond formation

时, 反应原料的对映选择性在手性转移过程中没有损 失.

2004 年 Toste 课题组进一步报道了使用 (dppm)$\mathrm{ReOCl}_{3}$ 催化芳烃化合物的炔丙基化反应 (Scheme 5, $b)^{[13]}$. 该反应采用富电子的芳烃作为亲核试剂与亲电试 剂炔丙醇反应, 将芳烃或杂芳烃的 $\mathrm{C}-\mathrm{H}$ 键直接转化为 $\mathrm{C}-\mathrm{C}$ 键. 此反应的唯一副产物是水, 因此提供了一条 构筑芳香化合物 $\mathrm{C}-\mathrm{C}$ 键的有效实用方法. 随后，作者 进一步将该方法应用于天然产物 $\beta$-阿朴鬼臼苦素的合 成中(Scheme 5, b).

Kuninobu 和 Takai 课题组 ${ }^{[14]}$ 在 2007 年报道了铼催 化的炔丙醇与有机硅试剂的偶联反应(图 6,a). 反应体 系使用 $\left[\operatorname{ReBr}(\mathrm{CO})_{3}(\mathrm{thf})\right]_{2}$ 催化炔丙基醇、茮基醇与烯丙 基、炔基硅烷反应生成新的 $\mathrm{C}-\mathrm{C}$ 键. 值得一提的是, 含

(a)

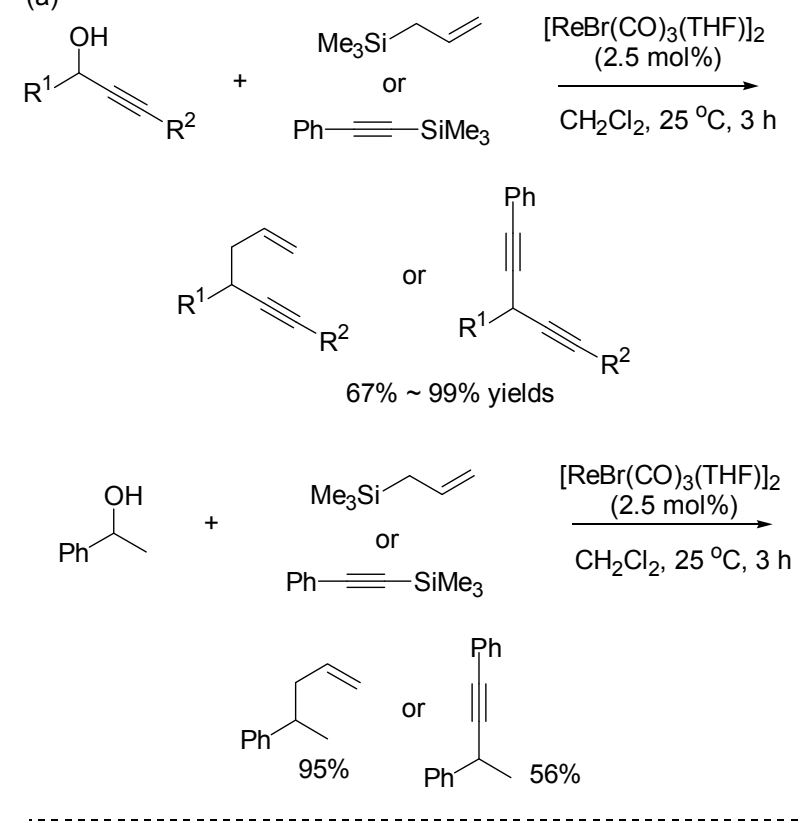

(b)

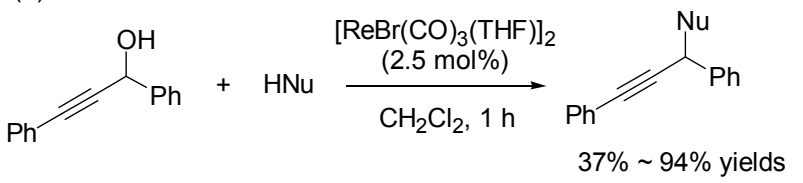

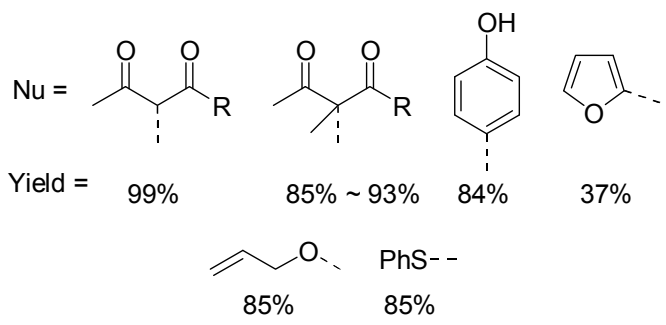

图式 $6\left[\operatorname{ReBr}(\mathrm{CO})_{3}(\mathrm{THF})\right]_{2}$ 催化炔丙醇、苄醇的脱羟基反应 构建新化学键

Scheme $6\left[\operatorname{ReBr}(\mathrm{CO})_{3}(\mathrm{THF})\right]_{2}$-catalyzed dehydroxylation reactions for new chemical bond formation 
有烷基官能团的炔丙基仲醇与有机硅试剂的脱羟基反 应一般是比较困难的, 然而在此反应体系中可以实现有 效的转化. 炔丙醇与炔基硅反应可以生成二炔基甲烷化 合物，这种化合物是制备高含碳材料的原料 ${ }^{[15]}$. 在此基 础上作者进一步发展了 $\left[\mathrm{ReBr}(\mathrm{CO})_{3}(\mathrm{THF})\right]_{2} / \mathrm{AuCl}$ 共催化 的芳香醛与炔基硅化合物的一锅煮反应制备二炔基甲 烷化合物.

2008 年, Kuninobu 和 Takai 课题组 ${ }^{[16]}$ 又报道了 $\left[\operatorname{ReBr}(\mathrm{CO})_{3}(\mathrm{THF})\right]_{2}$ 催化的炔丙基醇与其他亲核试剂脱 着基生成 $\mathrm{C}-\mathrm{C}$ 键的反应(Scheme $6, \mathrm{~b}$ ). 该反应体系对 于炔丙醇与 2,4-戊烷二酮的脱水偶联产物有优秀的产 率. 其他碳亲核试剂如 $\beta$-酮脂、苯酚、呋喃、吲哚都能 较好地与炔丙醇反应生成相应的 $\mathrm{C}-\mathrm{C}$ 键偶联产物. 在 反应体系中, 丙炔醇与氧、硫类亲核试剂, 例如乙醇、 烯丙醇、苯硫酚、十一硫醇等反应成功构筑了 $\mathrm{C}-\mathrm{O}$, $\mathrm{C}-\mathrm{S}$ 键. 作者认为可能的反应机理为: (1)通过铼促进的 脱羟基作用生成 2-丙炔基碳正离子; (2)亲核试剂亲核 进攻丙炔基碳正离子; (3)亲核进攻得到的反应中间体去 质子，生成偶联产物.

铼催化剂同样在催化单醇脱羟基生成烯烃, 构筑新 $\mathrm{C}-\mathrm{C}$ 反应中取得进展. 2010 年, Gebbink 课题组 ${ }^{[17]}$ 报道 $\mathrm{Re}_{2} \mathrm{O}_{7}$ 催化苄基醇脱羟基生成烯烃的反应. 该反应用 $\mathrm{Re}_{2} \mathrm{O}_{7}$ 作为催化剂, 以甲苯作为溶剂, 反应温度为 $100{ }^{\circ} \mathrm{C}$, 反应时间为 $24 \mathrm{~h}$. 在此反应体系下, 茮基仲醇 和叔醇生成烯烃具有优秀的产率(84\% 99\%) (Scheme $7, a)$. 在以上工作的基础上, Gebbink 等 ${ }^{[18]}$ 又将 $\mathrm{Re}_{2} \mathrm{O}_{7}$ 催 化反应体系应用于非苄基醇、萜类醇的脱羟基生成烯烃 的反应. 作者发现在反应中 $\mathrm{Re}_{2} \mathrm{O}_{7}$ 催化剂对烯丙基、烷 基、高烯丙基醇具有良好的脱羟基效果(Scheme 7,b). 另外, 萜类醇的脱羟基生成萜烯类产物的反应也具有优 秀的产率. 作者通过将 $\mathrm{Re}_{2} \mathrm{O}_{7}$ 与硫酸、一些固体酸做对 比展现出锞催化剂在脱羟基反应过程中具有良好的反 应活性和选择性.

\section{2 铼催化二(多元)醇脱羟基反应生成烯烃}

最近几年, 铼催化剂由于能催化二醇、多元醇和糖 等发生脱氧脱水反应(DODH)而受到化学家的广泛关注. 其具有底物适用范围广, 反应产率高, 反应区域选择性 和立体选择性好等优点. 铼催化二(多元)醇脱羟基反应 中通常使用三苯基膦、 $\mathrm{H}_{2}$ 、亚硫酸盐、醇或者元素单质 作为还原剂. 下文将基于不同的还原剂体系对近年来铼 催化的二(多元)醇脱羟基生成烯烃的研究进行介绍.

\section{1 三苯基膦作为还原剂}

1996 年, Andrews 课题组 ${ }^{[19]}$ 第一次报道了铼催化 的 DODH 反应 $($ Scheme $8, a)$. 反应体系使用 $\left(\mathrm{C}_{5} \mathrm{Me}_{5}\right)-$ (a)<smiles>CC(O)c1ccccc1</smiles>

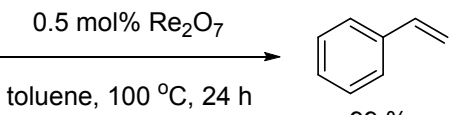

other examples:
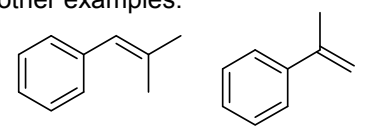

$99 \%$

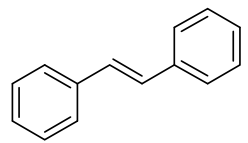

$99 \%$

$99 \%$ (cis:trans $=1: 13$ )

(b)

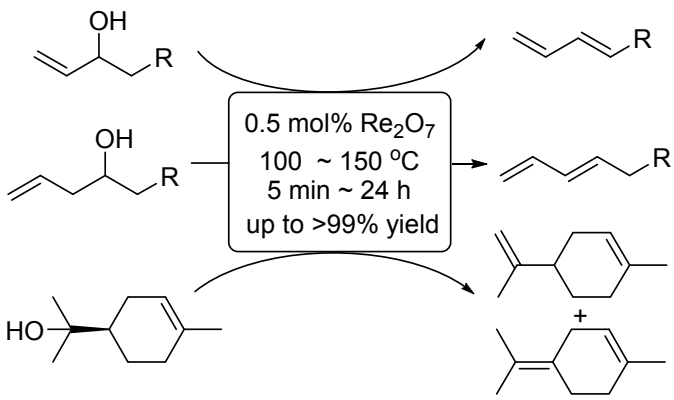

图式 $7 \mathrm{Re}_{2} \mathrm{O}_{7}$ 催化的单醇脱羟基反应

Scheme $7 \quad \mathrm{Re}_{2} \mathrm{O}_{7}$-catalyzed dehydroxylation reactions of monools

$\mathrm{ReO}_{3}$ 作为催化剂, 三苯基膦做为还原剂, 对于一些邻羟 基二醇和多元醇脱水脱氧生成烯烃具有良好的收率. 催 化循环包括三步(Scheme $8, \mathrm{~b}$ ): (1)三苯基膦还原脱除铼 催化剂中的一个氧; (2)二醇与脱掉一个氧的 $\mathrm{Cp} * \mathrm{ReO}_{2}$ 缩 合生成铼杂氧杂五元环中间体; (3)铼杂氧杂五元环中间 体中的 $\mathrm{C}-\mathrm{O}$ 键断裂生成烯烃. 该反应体系存在一定的 缺点, 例如反应速度较慢, 反应后生成当量的三苯基氧 化膦副产物, 并且需要缓慢的加入过量的三苯基膦来调 控催化剂体系，减少不脱羟基、过多的脱羟基等副反应 的发生.

2013 年 Gebbink 课题组 ${ }^{[20]}$ 报道了大位阻茂基三氧 化铼催化的邻羟基二醇生成烯烃的反应(Scheme $8, \mathrm{c}$ ). 该反应以三苯基膦作为还原剂，对于芳香族和脂肪族、 内部和终端的二醇生成烯烃都有优秀的产率 $(80 \%$ 95\%), 烯烃异构化产物少. 作者进一步将催化体系应用 于甘油醇、赤藓糖醇脱羟基的反应，发现甘油醇生成烯 丙醇有优秀的产率 $(91 \%)$, 但是赤藓糖醇在反应体系下 生成丁二烯和丁烯二醇的混合物只有中等产率. 当用醇 作还原剂的情况下，赤藓糖醇生成 1,3-丁二烯有 $67 \%$ 的 产率.

\section{$2.2 \mathrm{H}_{2}$ 作为还原剂}

$\mathrm{H}_{2}$ 作为绿色无污染的还原剂同样可以应用于二 (多)醇脱羟基生成烯烃的反应. 2009 年, Abu-Omar 课题 组 ${ }^{[21]}$ 报道了使用 $\mathrm{MeReO}_{3}$ (MTO)作为催化剂, $\mathrm{H}_{2}$ 作为 还原剂, 在温度 $150{ }^{\circ} \mathrm{C}$, 压力 $0.52 \sim 2.02 \mathrm{MPa}$ 条件下, 
(a)

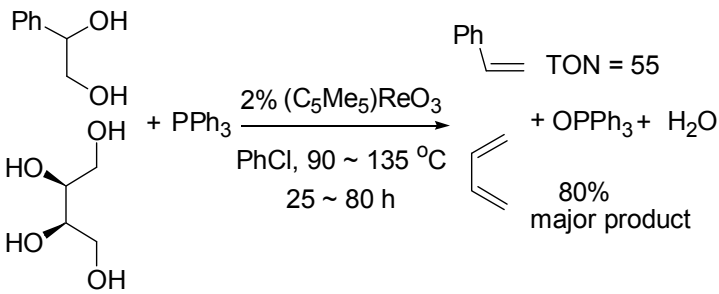

(b)

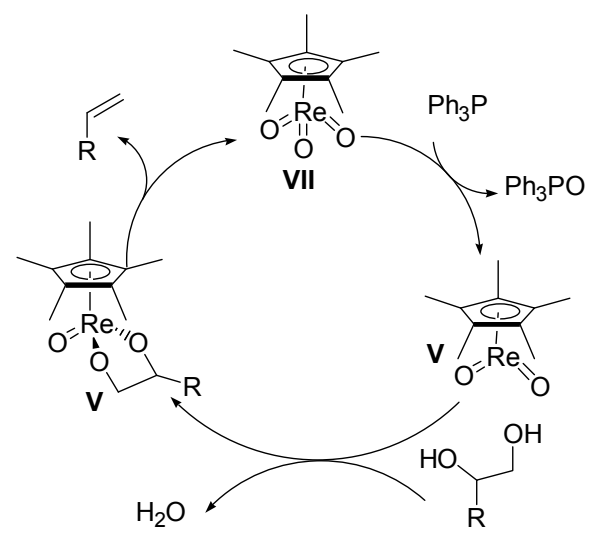

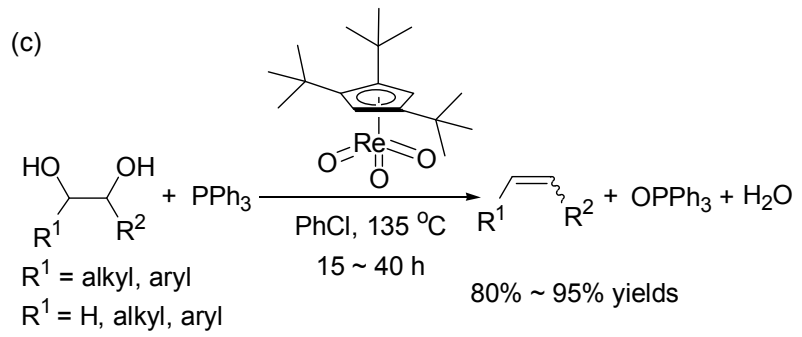<smiles>C=Cc1ccccc1</smiles>

图式 8 铼催化二(多)醇用三苯基磷作还原剂的脱羟基反应 Scheme 8 Rhenium-catalyzed dehydroxylation reactions of di(poly)ols using $\mathrm{Ph}_{3} \mathrm{P}$ as the reductant

实现环氧化合物(35\% 95\%)和二醇(18\% 60\%)的脱水 反应(Scheme 9, a). 高氢气压力下延长反应时间生成烷 烃主产物, 相对低的氢气压力下主要生成烯烃产物. 顺 式环状二醇(顺式-1,2-环己基二醇产率 60\%)脱羟基生成 相应烯烃比反式二醇的效率高, 可能是因为顺式环状二 醇更容易与铼催化剂形成铼氧杂环化合物的因素. 作者 认为催化循环如下 (Scheme 9, b): (1) $\mathrm{H}_{2}$ 还原 $\mathrm{MeReO}_{3}$ 形 成 $\mathrm{MeReO}_{2} \mathrm{~L}_{n}\left(\mathrm{~L}=\mathrm{THF}\right.$ 或 $\left.\mathrm{H}_{2} \mathrm{O}\right)$; (2) $\mathrm{MeReO}_{2} \mathrm{~L}_{n}$ 与二醇 缩合形成铼氧杂环化合物; (3) 铼氧杂环化合物脱除烯烃 完成催化循环. (a)

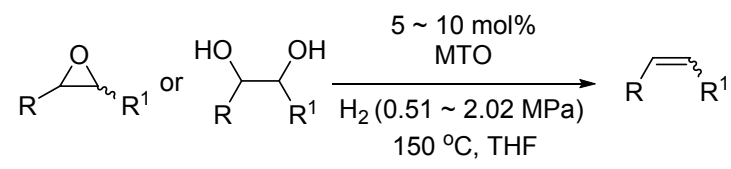

(b)

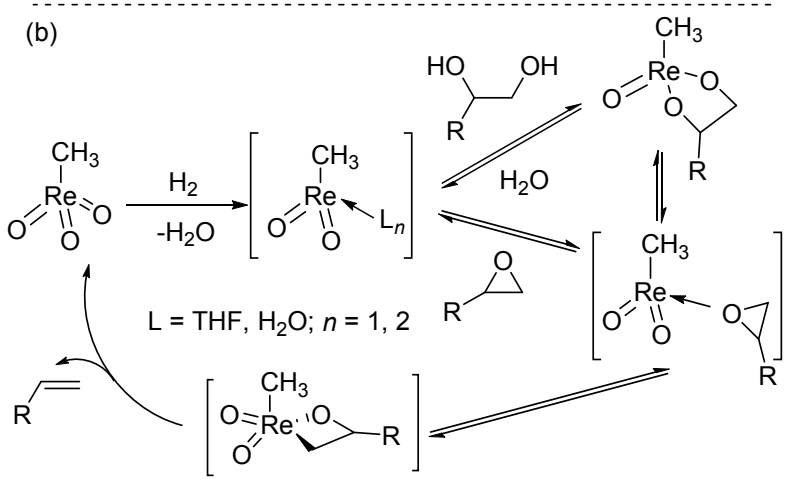

(c)
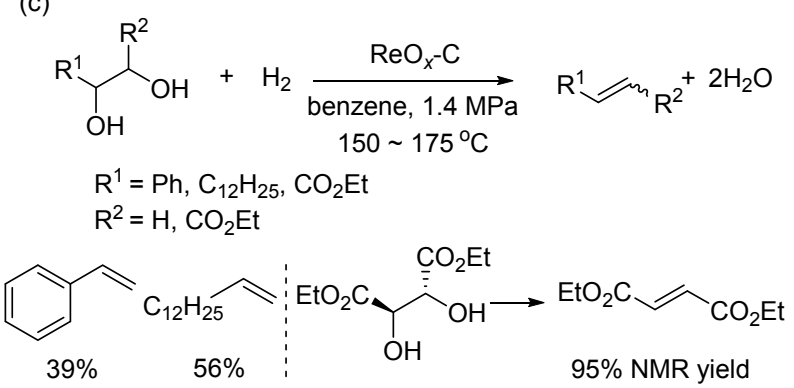

图式 9 铼催化二(多元)醇以 $\mathrm{H}_{2}$ 作还原剂的脱羟基反应 Scheme 9 Rhenium-catalyzed dehydroxylation reactions of di(poly)ols using $\mathrm{H}_{2}$ as the reductant

2013 年 Jentoft 课题组 ${ }^{[22]}$ 报道了用活性碳支持的高 铼酸盐作为催化剂, 使用 $\mathrm{H}_{2}$ 作为还原剂在高温条件下 $\left(150 \sim 170{ }^{\circ} \mathrm{C}\right)$ 实现的二醇脱羟基生成烯烃的反应 (Scheme 9, c). $\mathrm{ReO}_{x}$-C 催化剂是用水合 $\mathrm{NH}_{4} \mathrm{ReO}_{4}$ 通过平 衡吸附到活性碳上制备得到的，其中含有 3\% 4\%质量 分数的锞. 该反应体系对于苯乙二醇、1,2-正十四烷二 醇等都可以以中等收率得到相应的烯烃. $L-(+)$-酒石酸 二乙酯在此催化体系下可以高选择性地转化成反式的 富马酸二乙酯, 具有优秀的反应产率(95\%). 目前该反 应体系还存在催化剂(再循环利用)效率不高, 底物适用 范围窄等不足，有待于进一步改进.

\section{3 亚硫酸盐作为还原剂}

亚硫酸盐类化合物具有还原性能优秀 ${ }^{[23]}$ 、花费低、 使用方便、低毒、副产物硫酸盐可以回收 ${ }^{[24]}$ 等优点, 在 多元醇脱羟基反应中受到化学家们的较大关注. 2010 年, Nicholas 课题组 ${ }^{[25]}$ 首次报道了使用亚硫酸盐还原剂、 铼催化二醇在苯溶剂中脱羟基生成烯烃的反应. 作者使 用 $\mathrm{MeReO}_{3}$ 作为催化剂, $\mathrm{Na}_{2} \mathrm{SO}_{3}$ 作为还原剂的反应体系, 发现其对含芳香官能团的二醇脱羟基生成烯烃具有较 好的收率，然而在相同条件下含脂肪官能团的二醇反应 
效果不好, 烯烃产率低, 并且需要较长的反应时间 (Scheme 10,a). 如果反应体系采用没有溶剂、延长反应 时间等条件, 含脂肪官能团的二醇生成相应的烯烃有中 等的收率. 作者认为催化循环有两种可能途径(Scheme 10, b): (1) $\mathrm{MeReO}_{3}$ 与二醇缩合生成铼(VII)氧杂环化合 物, 然后, 亚硫酸盐还原铼(VII)氧杂环化合物生成铼 (V)氧杂环化合物; (2) $\mathrm{MeReO}_{3}$ 首先被亚硫酸盐还原生 成 $\mathrm{MeReO}_{2}$ 化合物, $\mathrm{MeReO}_{2}$ 与二醇缩合形成铼 $(\mathrm{V})$ 氧杂 环化合物. 最后, 铼 $(\mathrm{V})$ 氧杂环化合物发生消除即得到 烯烃产物.

(a)

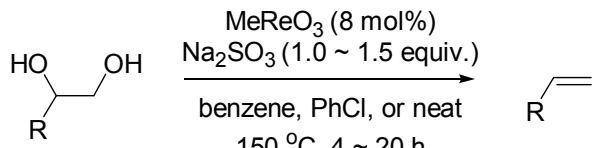

$\mathrm{R}=\mathrm{Ph}$, alkyl $(\mathrm{C}>6)$ $150^{\circ} \mathrm{C}, 4 \sim 20 \mathrm{~h}$ up to $60 \%$ yield

(b)

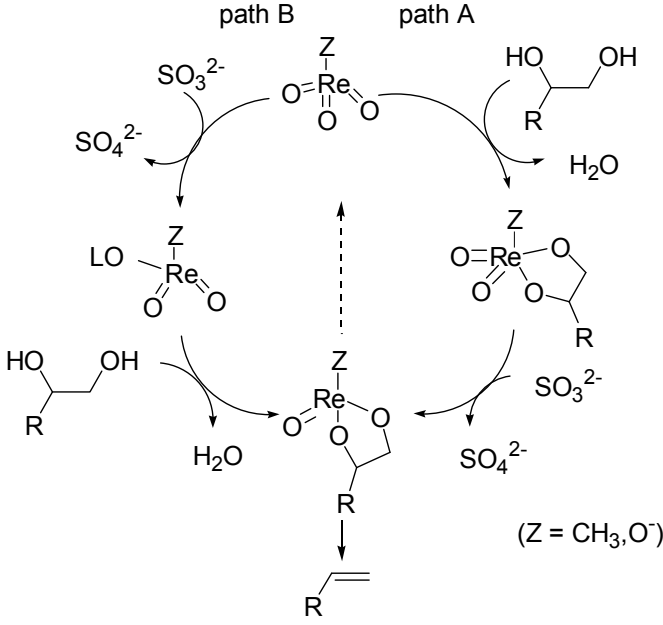

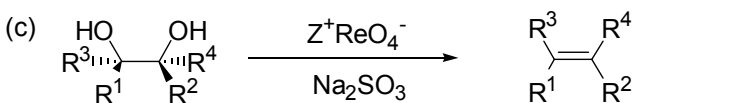

$$
\begin{aligned}
& \mathrm{R}^{1}=\text { alkyl, aryl } \mathrm{R}^{2}=\mathrm{R}^{3}=\mathrm{R}^{4}=\mathrm{H} \\
& \mathrm{R}^{1}=\mathrm{R}^{2}=\text { carbocycle; } \mathrm{R}^{3}=\mathrm{R}^{4}=\mathrm{H} \\
& \mathrm{R}^{1}=\mathrm{R}^{4}=\mathrm{CO}_{2} \mathrm{R} ; \mathrm{R}^{2}=\mathrm{R}^{3}=\mathrm{H}
\end{aligned}
$$

图式 10 铼催化二(多元)醇以亚硫酸盐作还原剂的脱羟基反 应

Scheme 10 Rhenium-catalyzed dehydroxylation reactions of di(poly)ols using sulfite as the reductant

2011 年, Nicholas 课题组 ${ }^{[26]}$ 在以前工作的基础上继 续探索铼催化，亚硫酸盐作还原剂二醇的脱羟基生成烯 烃反应. 作者探寻了多元醇底物、还原剂、催化剂、溶 剂及各种添加剂对反应的影响. 发现 $\mathrm{MeReO}_{3}$ 催化, $\mathrm{Na}_{2} \mathrm{SO}_{3}$ 作还原剂的体系, 对于二醇脱羟基生成烯烃反 应有好的活性, $\mathrm{Z}^{+} \mathrm{ReO}_{4}^{-}\left(\mathrm{Z}=\mathrm{Na}, \mathrm{Bu}_{4} \mathrm{~N}\right)$ 作催化剂时体系 相对具有好的选择性(Scheme 10, c). 反应需要的时间很 长, 一些反应需 $100 \mathrm{~h}$ 或者更多的反应时间, 作者发现 加入相转移剂 15-冠醚-5 有助于还原剂的转化, 烯烃产
率的提高, 并且能够缩短反应的时间. 反应体系选择性 地转化顺式 1,2-环己基二醇脱羟基生成相应烯烃(25\%), $L-(+)$-酒石酸二乙酯转化成反式富马酸二乙酯 $(35 \%)$.

\section{4 醇作为还原剂}

在铼催化的二醇脱着基生成烯烃的反应中，对环境 友好的醇也可以作为一种有效的还原剂. 2010 年, Ellman 和 Bergman 课题组 ${ }^{[27]}$ 首次报道了用 $\operatorname{Re}_{2}(\mathrm{CO})_{10}$ 催 化, 使用仲醇作为还原剂, 在氧气条件下, 邻羟基二醇 脱羟基生成烯烃的反应(Scheme 11,a). 该反应适用于端 位、内部取代的邻羟基二醇脱羟基生成烯烃，反应产率 较高 $(50 \% \sim 84 \%)$. 反应后，作为还原剂的单醇生成相 应的酮副产物. 在反应体系中加入催化量的 $\mathrm{TsOH}$ 或 $\mathrm{H}_{2} \mathrm{SO}_{4}$ 能降低反应温度, 减少反应时间, 降低催化剂的 用量, 并且能提高烯烃产物的产率. 采用该反应体系, 3,4-癸烷二醇高选择性的生成反式-3-癸烯，表明在二醇 脱羟基生成烯烃过程中存在顺式消除. 顺式-1,2-环己基 二醇在反应体系中较好的生成环己烯 $(72 \%)$, 然而反式 的 1,2-环己基二醇没有反应. 作者将此反应体系进一步 应用于具有生物活性的赤藓糖醇的脱羟基反应，发现反 应后生成 2,5-二氢呋喃产物(62\%).

(a)
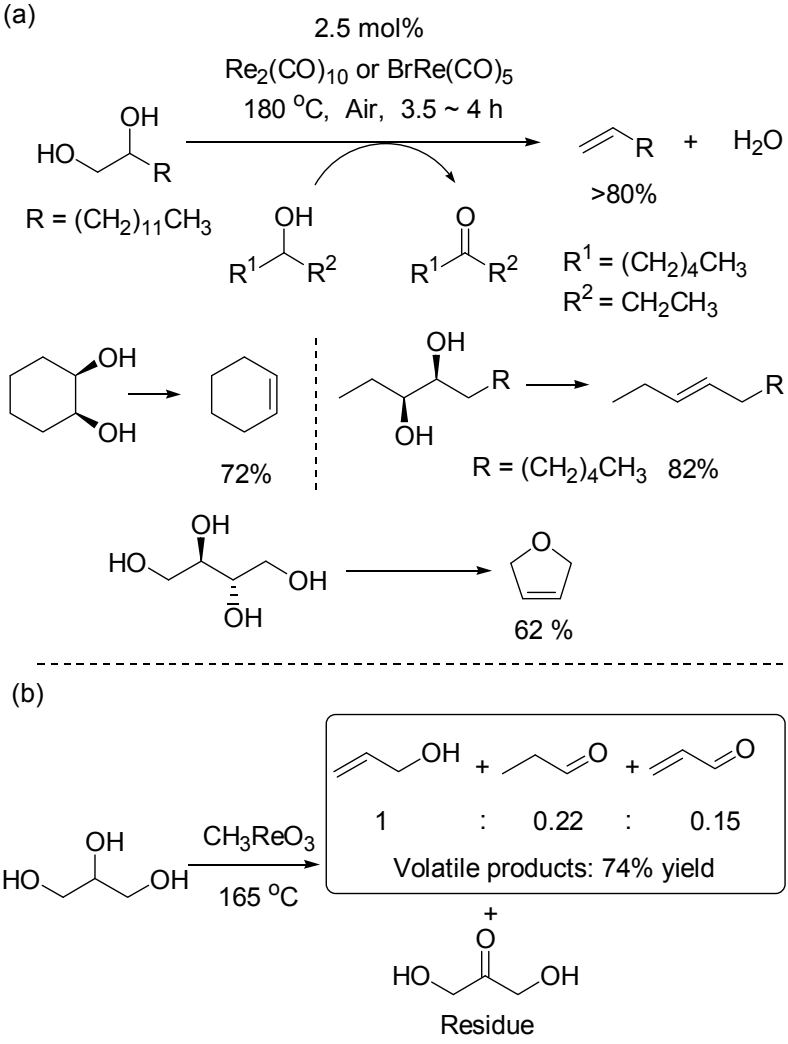

图式 11 铼催化二(多元)醇以醇作还原剂的脱羟基反应 Scheme 11 Rhenium-catalyzed dehydroxylation reactions of di(poly)ols using alcohols as reductants

2012 年, Abu-Omar 课题组 ${ }^{[28]}$ 报道了铼催化甘油醇 
的脱羟基反应. 反应用 $\mathrm{MeReO}_{3}$ 作为催化剂, 甘油醇作 为底物和还原剂, 在 $165{ }^{\circ} \mathrm{C}$ 温度下, 甘油醇转化成烯丙 醇为主的挥发性产物, 氧化产物二羟基丙酮由于低蒸气 压残留在反应血中(Scheme 11, b). 反应扩大量后可通 过调整温度蒸馏提取出生成的产物. 该反应体系适用于 生物内消旋赤藓糖醇, 生成 $58 \%$ 产率的二氢呋喃. 另外, 此反应展现出良好的立体选择性, 顺式-1,2-环己基二醇 在反应体系中有 $91 \%$ 的产率生成环已烯, 然而反式-1,2环己基二醇则没有反应.

2012 年, Toste 课题组 ${ }^{[29]}$ 报道了用仲醇 $(\mathrm{C}>5)$ 作还 原剂和溶剂, $\mathrm{MeReO}_{3}$ 作催化剂催化糖和糖醇的脱羟基 反应(Scheme 12, a). 该反应体系底物适用范围广, 能将 复杂的糖和糖醇顺利转化成线性链烯烃和芳烃, 具有显 著的选择性和产率. 反应体系适用于 $\mathrm{C}_{3}$ 的甘油醇和 $\mathrm{C}_{4}$ 的赤藓糖醇脱羟基反应, 以优秀的产率生成相应产物烯 丙醇( $90 \%$ ) 和 1,3-丁二烯( $81 \%$ ). $C_{5}$ 的糖醇如木糖醇、核 糖醇在此反应体系下脱羟基与作为还原剂的醇发生醚 化反应, 生成 $E-2,4$-烯丙基-3-戊基醚(33\% 61\%). $\mathrm{C}_{6}$ 糖 醇如山梨醇、甘露醇脱羟基则生成 $E$-已三烯 $(54 \%)$. 具 有立体构型的纤维醇以中等收率生成苯与苯酚的混合 物, 赤藓糖、苏阿糖则生成呋喃化合物 $(47 \% \sim 60 \%)$. 己 糖生成乙烯呋喃和呋喃产物, 但是在目前条件下产率不 高. 作者认为反应机理是(Scheme 12, b): (1) $\mathrm{Re}(\mathrm{VII}) \mathrm{O}_{3}$ 被醇还原生成 $\operatorname{Re}(\mathrm{V}) \mathrm{O}_{2}$ 化合物, (2) $\mathrm{Re}(\mathrm{V}) \mathrm{O}_{2}$ 化合物与二 醇结合生成钭二氧杂环中间体, (3) 钭二氧杂环中间体脱 除烯烃, 生成 $\mathrm{CH}_{3} \mathrm{ReO}_{3}$ 完成催化循环.

Toste 课题组 ${ }^{[30]}$ 在前期工作的基础上, 继续探索锞 催化以醇作为还原剂的二醇(多元醇)的脱羟基反应. 作 者 ${ }^{[31]}$ 利用 $\mathrm{MeReO}_{3}$ 促使烯丙醇发生[1,3]-OH 转移, 然后 发生脱羟基反应从而首次实现了二醇的 1,4-和 1,6-脱水 脱氧生成相应的烯烃(Scheme 13, a). 另外, 作者在前面 工作的基础上进一步拓展了多元醇的底物适用范围. 在 催化剂 $\mathrm{HReO}_{4}$ 的催化下, 正丁醇作为溶剂和还原剂, $\mathrm{C}_{6}$ 糖酸、半乳糖二酸及其酯衍生物转化成烯烃酯化物, 具有较好的产率 $(71 \%)$. 同样, $\mathrm{C}_{6}$ 葡萄糖酸可以有效 地转化成 $E, E$-二烯烃酯化合物( $(47 \%)$.

2013 年, Nicholas 课题组 ${ }^{[32]}$ 报道了 $\mathrm{NH}_{4} \mathrm{ReO}_{4}$ 催化, 贲醇作为还原剂的二醇脱羟基生成不饱和烯烃的反应 (Scheme 13, b). 该反应体系能将多种二醇有效地转化 成相应的烯烃产物, 具有较好的产率 $(50 \% \sim 95 \%)$. 反 应的副产物是苯甲醛, 反应后与烯烃容易分离, 催化剂 重新回收利用仍然有较高的活性.

\section{5 元素( $\mathrm{Zn}, \mathrm{Fe}, \mathrm{Mn}, \mathrm{C})$ 作为还原剂}

为了寻找钭催化醇脱羟基反应的新型、有效、可回 收的还原剂, Nicholas 课题组将研究的焦点转移到零价 (a)

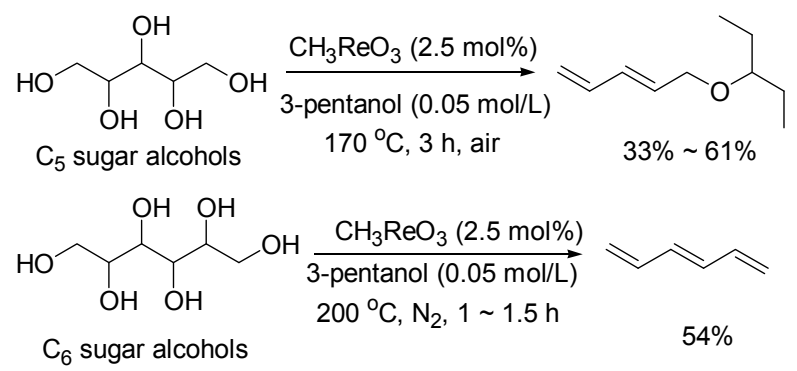

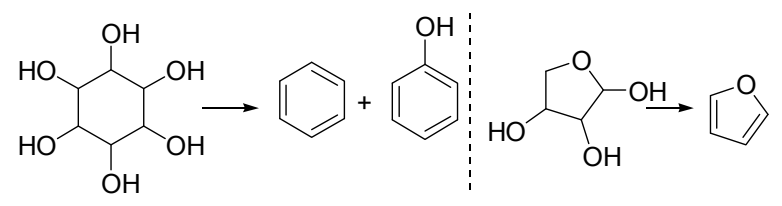

inositols to aromatics $17 \% \sim 64 \%$

tetroses to furan $47 \% \sim 60 \%$

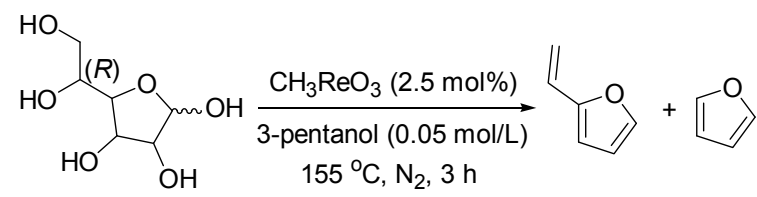

hexoses to 2-vinylfuran and furan $\quad 25 \% \sim 40 \%$

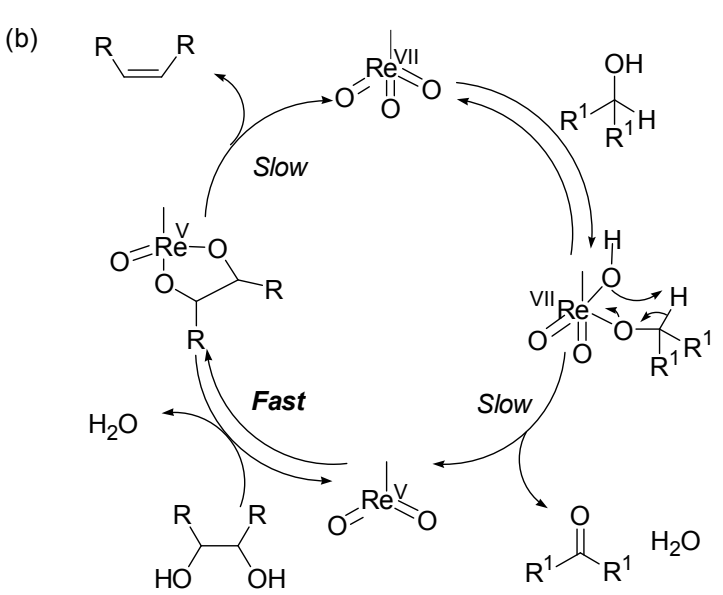

图式 12 铼催化糖和糖醇以仲醇作还原剂的脱羟基反应 Scheme 12 Rhenium-catalyzed dehydroxylation reactions of sugars and sugar alcohols using secondary alcohols as reductants

元素单质作还原剂上来. 2014 年, 该课题组 ${ }^{[33]}$ 首次报道 了使用 $\mathrm{Zn}, \mathrm{Fe}, \mathrm{Mn}, \mathrm{C}$ 单质作还原剂, 实现了钭催化的 二醇脱羟基生成烯烃的反应(图 14). 元素单质还原剂使 用简单、方便，价格便宜，反应后生成的氧化物与产物 烯烃容易分离, 有利于回收再应用. 作者使用元素 Zn, $\mathrm{Fe}, \mathrm{Mn}, \mathrm{C}$ 单质作为还原剂, $\mathrm{NH}_{4} \mathrm{ReO}_{4}$ 催化 1,2-癸烷二醇 生成相应的烯烃有较好的收率. 在反应体系条件下, 如 果使用 $\mathrm{Zn}$ 单质作为还原剂, 阳离子铼 $(\mathrm{V})\left[(\mathrm{Py})_{4} \mathrm{ReO}_{2}\right] \mathrm{Cl}$ 作为催化剂, 1,2-癸烷二醇生成烯烃有 $90 \%$ 的收率. 在 $\mathrm{NH}_{4} \mathrm{ReO}_{4}$ 催化体系下, 酒石酸二乙酯生成反式的富马酸 
(a)

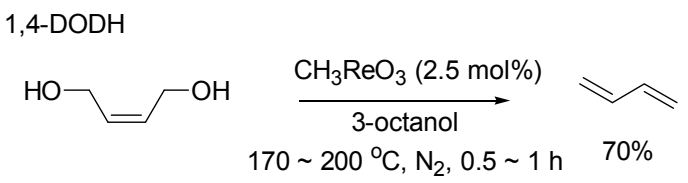

$1,6-\mathrm{DODH}$
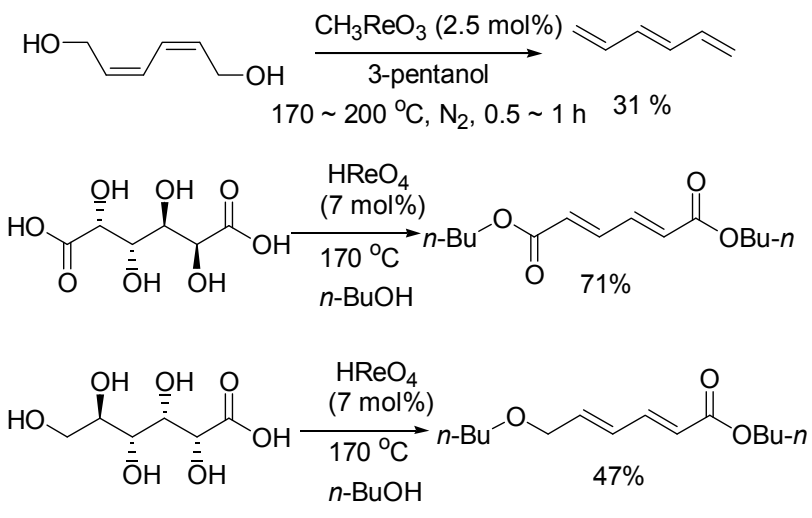

(b)

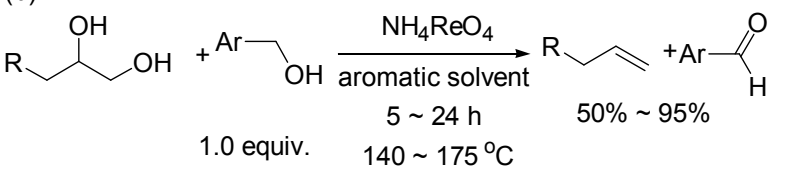

$\mathrm{R}=$ alkyl, ether, ester, $\mathrm{OH}$

图式 13 铼催化二(多元)醇以醇作还原剂的脱羟基反应扩展 Scheme 13 Expansion of rhenium-catalyzed dehydroxylation reactions of di(poly)ols using alcohols as reductants

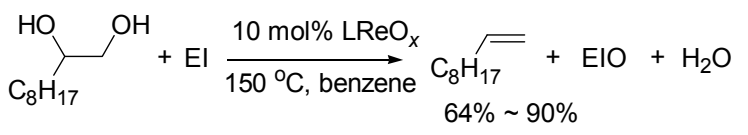

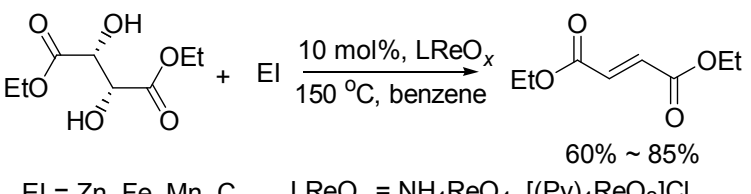

图式 14 铼催化二(多元)醇以元素单质作还原剂的脱羟基反 应

Scheme 14 Rhenium-catalyzed dehydroxylation reactions of di(poly)ols using elemental reductants

二乙酯 $(65 \% \sim 85 \%)$. 作者认为反应机理为: 铼(V)催化 剂 $\left[(\mathrm{Py})_{4} \mathrm{ReO}_{2}\right]^{+}$与醇缩合生成铼 $(\mathrm{V})$ 二氧杂环中间体, 其 脱除烯烃生成 $\left(\left[(\mathrm{Py})_{4} \operatorname{Re}(\mathrm{VII}) \mathrm{O}_{3}\right]^{+}\right)$化合物, 元素单质还 原剂通过还原脱除铼(VII)化合物中的氧重新生成铼(V) 催化剂, 从而完成催化循环.

\section{3 总结与展望}

在全球资源危机的大背景下, 发展新型、高效的方 法将丰富的可再生资源转化成燃料和化学品, 符合当前 社会可持续发展的迫切要求. 铼催化体系在单醇、二醇
及多元醇类化合物的脱羟基反应中的研究在近年来取 得了重要的进展，此类反应具有选择性好，反应条件简 单，反应产率高等优点. 但是，铼催化的醇类化合物的 脱羟基反应还存在催化效率低，反应温度较高，反应类 型较少、反应底物适用范围较窄等局限性。如果可以利 用自然界或工业界来源广泛的糖类化合物，例如葡萄糖 二酸(glucaric acid), 解决铼催化剂对此类特定底物的适 用性，在未来实现铼催化的葡萄糖二酸脱羟基反应生成 己二酸的精细化工转化等问题，必将产生重要的学术价 值和社会应用价值. 可喜的是, Toste 课题组已经在这个 方面取得了一定的进展(Scheme 13,a). 今后，发展新型 的铼催化剂, 特别是配体调控的高效铼催化剂并深入研 究其反应性可能是解决这些问题的突破口，相信未来会 取得较大的发展.

\section{References}

[1] (a) Cao, X. H. Chem. Ind. Eng. Prog. 2007, 26,905 (in Chinese). (曹湘洪，化工进展, 2007, 26, 905.)

(b) Min, E. CIESC J. 2006, 57, 1739 (in Chinese)

(闵恩泽，化工学报, 2006, 57,1739 .)

(c) Vennestrom, P. N. R.; Osmundsen, C. M.; Christensen, C. H.; Taarning, E. Angew. Chem., Int. Ed. 2011, 50, 10502.

[2] Ragauskas, A. J.; Williams, C. K.; Davison, B. H.; Britovsek, G.; Cairney, J.; Eckert, C. A.; Frederik, W. J. Jr.; Hallett, J. P.; Leak, D. J.; Liotta, C. L.; Mielenz, J. R.; Murphy, R.; Templer, R.; Tschaplinski, T. Science 2006, 311, 484.

[3] Werpy, T.; Peterson, G. Top Value Added Chemicals from Biomass: Vol. 1, U. S. Department of Energy, Washington D. C., 2004.

[4] Corma, A.; Iborra, S.; Velty, A. Chem. Rev. 2007, 107, 2411.

[5] For our work, see: (a) Xia, D.; Wang, Y.; Du, Z.; Zheng, Q.-Y.; Wang, C. Org. Lett. 2012, 14, 588

(b) Wang, Y.; Zhang, L.; Yang, Y.; Zhang, P.; Du, Z.; Wang, C. J. Am. Chem. Soc. 2013, 135, 18048.

(c) Tang, Q.; Xia, D.; Jin, X.; Zhang, Q.; Sun, X.-Q.; Wang, C. J. Am. Chem. Soc. 2013, 135, 4628 .

(d) Tang, H.; Zhou, B.; Huang, X.-R.; Wang, C.; Yao, J.; Chen, H. ACS Catal. 2014, 4, 649.

For selected work from other groups, see: (e) Kuninobu, Y.; Takai, K. Chem. Rev. 2011, 111, 1938.

(f) Jin, H.; Xie, J.; Pan, C.; Zhu, Z.; Cheng, Y.; Zhu, C. ACS Catal. 2013, 3, 2195.

(g) Fukumoto, Y.; Daijo, M.; Chatani, N. J. Am. Chem. Soc. 2012 134,8762

(h) Hua, R.; Tian, X. J. Org. Chem. 2004, 69, 5782.

(i) Liu, Q.; Li, Y.-N.; Zhang, H.-H.; Chen, B.; Tung, C.-H.; Wu, L.-Z. J. Org. Chem. 2011, 76, 1444.

[6] Zhu, Z.; Espenson, J. H. J. Org. Chem. 1996, 61, 324.

[7] Sherry, B. D.; Radosevich, A. T.; Toste, F. D. J. Am. Chem. Soc 2003, 125, 6076 .

[8] Liu, Y.; Hua, R. M.; Sun, H. B.; Qiu, X. Q. Organometallics 2005 , 24, 2819.

[9] Ohri, R. V.; Radosevich, A. T.; Hrovat, K. J.; Musich, C.; Huang, D.; Holman, T. R.; Toste, F. D. Org. Lett. 2005, 7, 2501.

[10] Abdukader, A.; Jin, H.; Cheng, Y.; Zhu, C. Tetrahedron Lett. 2014, 55,4172 .

[11] Xu, Q.; Li, Q. Chin. J. Org. Chem. 2013, 33, 18 (in Chinese). 
(徐清, 李强, 有机化学, 2013, 33, 18.)

[12] Luzung, M. R.; Toste, F. D. J. Am. Chem. Soc. 2003, 125, 15760.

[13] Kennedy-Smith, J. J.; Young, L. A.; Toste, F. D. Org. Lett. 2004, 6, 1325 .

[14] Kuninobu, Y.; Ishii, E.; Takai, K. Angew. Chem., Int. Ed. 2007, 46, 3296.

[15] Nielsen, M. B.; Diederich, F. Synlett 2002, 544.

[16] Kuninobu, Y.; Ueda, H.; Takai, K. Chem. Lett. 2008, 8, 878.

[17] Korstanje, T. J.; Jastrzebski, J. T. B. H.; Klein Gebbink, R. J. M. ChemSusChem 2010, 3, 695.

[18] Korstanje, T. J.; Waard, E. F.; Jastrzeki, J. T. B. H.; Klein Gebbink, R. J. M. ACS Catal. 2012, 2, 2173.

[19] Cook, G. K.; Andrews, M. A. J. Am. Chem. Soc. 1996, 118, 9448.

[20] Raju, S.; Jastrzebski, J. T. B. H.; Robertus, M. L.; Klein Gebbink, R. J. M. ChemSusChem 2013, 6, 1673.

[21] Ziegler, J. E.; Zdilla, M. J.; Evans, A. J.; Abu-Omar, M. M. Inorg. Chem. 2009, 48, 9998.

[22] Denning, A. L.; Dang, H.; Liu, Z. M.; Nicholas, K. M.; Jentoft, F. C. ChemCatChem 2013, 12, 3567 .

[23] (a) Holm, R. H.; Donahue, J. P. Polyhedron 1993, 12, 571. (b) Lee, S. C.; Holm, R. H. Inorg. Chim. Acta 2008, 361, 1166.
[24] Kaksonen, A. H.; Puhakka, J. A. Eng. Life Sci. 2007, 7, 541.

[25] Vkuturi, S.; Chapman, G.; Ahmad, I.; Nicholas, K. M. Inorg. Chem. 2010, 49, 4744.

[26] Ahmad, I.; Chapman, G.; Nicholas, K. M. Organometallics 2011, $30,2810$.

[27] Arceo, E.; Ellman, J. A.; Bergman, R. G. J. Am. Chem. Soc. 2010, 132,11408

[28] Yi, J.; Liu, S.; Abu-Omar, M. M. ChemSusChem 2012, 5, 1401.

[29] Shiramizu, M.; Toste, F. D. Angew. Chem., Int. Ed. 2012, 51, 8082.

[30] Shiramizu, M; Toste, F. D. Angew. Chem., Int. Ed. 2013, 52, 12905.

[31] For selected transformations, see: (a) Bellemin-Laponnaz, S. ChemCatChem 2009, 1, 357.

(b) Morrill, C.; Grubbs, R. H. J. Am. Chem. Soc. 2005, 127, 2842.

(c) Morrill, C.; Beutner, G. L.; Grubbs, R. H. J. Org. Chem. 2006, 71,7813 .

(d) Hansen, E. C.; Lee, D. J. Am. Chem. Soc. 2006, 128, 8142.

(e) Herrmann, A. T.; Saito, T.; Stivala, C. E.; Tom, J.; Zakarian, A. J. Am. Chem. Soc. 2010, 132, 5962.

[32] Jacobs, C. B.; Nicholas, K. M. ChemSusChem 2013, 6, 597.

[33] McClain, J. M.; Nicholas, K. M. ACS Catal. 2014, 4, 2109.

(Zhao, X.) 